\title{
Pour un usage raisonné de la numérisation de l'enquête ethnographique
}

Thomas Cornillet et Caroline Datchary

\section{OpenEdition}

\section{Journals}

Édition électronique

URL : http://journals.openedition.org/pa/947

DOI : $10.4000 /$ pa. 947

ISSN : 2273-0362

Éditeur

Université Lumière Lyon 2

\section{Édition imprimée}

Date de publication : 28 juillet 2020

Pagination : 40-57

ISSN : 1634-7706

\section{Référence électronique}

Thomas Cornillet et Caroline Datchary, « Pour un usage raisonné de la numérisation de l'enquête ethnographique », Parcours anthropologiques [En ligne], 15 | 2020, mis en ligne le 20 juillet 2020, consulté le 10 décembre 2020. URL : http://journals.openedition.org/pa/947 ; DOI : https://doi.org/ 10.4000/pa.947 


\section{Pour un usage raisonné de la numérisation de l'enquête ethnographique}

\section{Thomas Cornillet}

LISST-CERS, Université de Toulouse 2 Jean-Jaurès

\section{Caroline Datchary}

LISST-CERS, Université de Toulouse 2 Jean-Jaurès

\section{INTRODUCTION}

Les relations entre numérique et anthropologie sont évidemment plurielles. Tout d'abord, le développement des dispositifs numériques offre la possibilité d'une anthropologie du numérique, ou cyberanthropologie - pour reprendre le terme proposé par Escobar dès 1994 (Escobar, 1994). Nous pensons qu'il y a un réel danger à réifier les mondes numériques: de fait les mondes se numérisent de plus en plus et cette numérisation doit être mise à l'agenda de l'anthropologue, mais pour être pleinement analysée, elle ne doit toutefois pas être artificiellement coupée des activités non numérisées de ces mondes (Dagiral et Martin, 2016; Pastinelli, 2011). Plus spécifiquement, c'est la question de la numérisation de l'activité de l'ethnographie en elle-même qui retiendra ici notre attention, soit plus une question de méthode que d'objet. Cette numérisation concerne les trois différents moments de l'enquête ethnographique : la réalisation du terrain, son analyse et la publication de la recherche. Les deux premiers moments sont évidemment étroitement liés et peuvent être difficilement traités séparément. Nous choisissons ici de ne pas traiter la questions de la diffusion électronique et numérique des savoirs anthropologiques, question abordée dans la littérature scientifique depuis longtemps maintenant (Schwimmer, 1996) et qui fait l'objet de nombreuses contributions régulières (Casilli, 2014 ; Dupré, Walliser, Lévy, 2011 ; Murillo, 2018) pointant les opportunités et les limites, notamment en matière d'éthique, de rapports de pouvoir mais aussi concernant la qualité souvent inégale des ressources mises à disposition en ligne.

Mais les outils numériques, loin de constituer un profond bouleversement, ne sont-ils qu'une médiation de plus de l'enquête ethnographique ? C'est en tout cas, à l'instar d'autres chercheurs et chercheuses tels que Dominique Boullier (2016), le parti que nous prenons et que nous développerons dans une première partie, avant d'examiner dans un second temps les promesses et les limites de la numérisation de l'enquête. Enfin, à travers les choix 
méthodologiques concrets réalisés dans le cadre d'une enquête sur la communication au travail, nous montrerons l'intérêt à hybrider méthodes traditionnelles et numériques.

\section{LE NUMÉRIQUE, UNE MÉDIATION SUPPLÉMENTAIRE DU TRAVAIL D'ENQUÊTE}

Quels effets peut avoir la numérisation sur le travail d'enquête ethnographique? La numérisation de nos sociétés, bien que rapide, massive et transverse à tous les mondes sociaux, ne constitue pas une révolution, mais bien une transformation technique de grande ampleur dont il convient de saisir les incidences en termes $\mathrm{d}^{\prime}$ " amplification» et de «transformation propre » (Boullier, 2016). Dès lors, pour mieux distinguer ces effets propres ou amplificateurs de la numérisation, il convient de s'interroger au préalable sur les différentes médiations, y compris non numériques, qui équipent le travail d'enquête ethnographique: celles-ci sont à la fois corporelles, scripturales et techniques.

Le corps constitue évidemment une indispensable médiation, via la perception mais aussi les affects. En effet, Jeanne Favret-Saada a bien montré suite à son enquête sur la sorcellerie dans le bocage, comment la connaissance ne peut se passer des affects (1990). Pierre Livet quant-à-lui s'est attaché à montrer le rôle des émotions comme "proto-évaluation » ou traitement primaire de l'environnement (1998). Le lien entre le corps et la cognition est encore plus patent au regard de nos capacités perceptives. Les travaux d'Ingold autour de l'écologie de la perception (2013) insistent sur la description des ressorts cognitifs et pratiques de l'action; on retrouve là tout le courant de la cognition distribuée, voire de l'action située.

Prenons l'exemple de la perception visuelle qui constitue un médium corporel dominant de l'enquête ethnographique, bien que non exclusif (voir par exemple Candau et Jeanjean, 2006). Les travaux de Charles Goodwin sur la « vision professionnelle » (Goodwin et Goodwin, 1996) ont mis en évidence que les compétences visuelles étaient propres à chaque communauté de travail. La transmission des compétences visuelles par les membres les plus expérimentés d'une communauté est indissociable des "méthodes" (Garfinkel, 1986) par lesquelles ils et elles construisent du sens autour de ce qu'ils et elles voient. Dans le cas de l'ethnographe, cette compétence est assurément centrale, notamment la capacité à discriminer, dans son environnement de travail, les détails pertinents de ceux qui ne le sont pas (Datchary, 2013).

L'ethnographe "classique » s'équipe d'un carnet de terrain sur lequel il ou elle écrit ce qu'il ou elle voit. Envisageons donc maintenant cette médiation supplémentaire dans le travail d'enquête que constitue l'écriture. Jack Goody montre bien les conséquences dans la construction des savoirs de l'usage de 
l'écriture (1979) : par exemple sur les sociétés orales, le recours à l'écriture va figer un mythe dans une version donnée et gommer les conditions d'énonciation. De même, la mise en tableau des systèmes de classification attribués à une société sans écriture va introduire des distorsions, car elle induit une logique binaire inadéquate, biais vers une rationalisation indue et un systématisme contestable. La prise de notes de l'ethnographe participe donc pleinement au processus de structuration des idées et de la pensée, comme en attestent les nombreux travaux d'anthropologues et de sociologues consacrés à la question depuis longtemps maintenant ${ }^{1}$.

Si les médiations corporelles et scripturales sont centrales, les médiations techniques se développent de plus en plus et affectent en retour les pratiques corporelles. La vision de l'ethnographe est de plus en plus équipée d'appareils de prise de vue, voire parfois d'artefacts plus sophistiqués à l'instar des oculomètres (Grimaud, 2013). Les "manières de voir » ne sont plus alors les mêmes, les compétences cognitives se transformant en fonction de l'équipement artefactuel, comme cela a été montré dans le cas du pilote d'avion et de son cockpit (Hutchins, 1994) ou du trader et du marché financier «aprésenté » par son écran (Knorr Cetina et Bruegger, 2002). Lorsque l'équipement artefactuel de la vision vient à se modifier, ces façons de voir doivent être adaptées. Dès lors que le terrain n'est plus capté visuellement et retranscrit dans un carnet, mais capturé directement sur un écran, là encore cette médiation n'est pas neutre cognitivement. Par exemple, en restituant tout ou partie du terrain de manière non scripturale, le chercheur ou la chercheuse peut revenir autant de fois que nécessaire sur ces données pour investiguer des éléments qu'il ou elle n'avait pas forcément en tête au moment du recueil ; cela offre également la possibilité de réaliser des analyses collectives sur des terrains que l'on a réalisés en partie voire pas du tout, à l'instar des data sessions sur les données vidéos des ethnométhodologues.

Les débats méthodologiques en anthropologie et sociologie visuelle autour des effets de la médiation technique, de la photographie à la miniaturisation des caméras numériques, en témoignent (Becker, 2001 ; Colleyn, 2012). Ils pointent, entre autres, la nécessité pour les chercheurs et chercheuses de se former aux techniques cinématographiques afin de ne pas oublier le travail interprétatif qu'ils et elles opèrent de manière plus ou moins consciente à travers l'acte de filmer. Sylvie Grosjean, Florian Grandena et Luc Bonneville le résument très bien: "en faisant usage de la caméra, le chercheur a l'obligation, en quelque sorte, d'être conscient de ses choix et du travail de " construction " qu'il effectue conséquemment sur le « réel » (2017, en ligne). Cet équipement affecte également la médiation scripturale : l'évolution des technologies des captations en Analyse Conversationnelle a de fait transformé les conventions de transcriptions (Relieu, 2013). Elle a aussi déplacé

\footnotetext{
${ }^{1}$ Voir notamment, le numéro 111-12 de la revue L'Homme, "L'écriture des sciences de l'homme », paru en 1989, et le numéro 58 de la revue Communication paru en 1994 ainsi que l'article de Jérôme Denis et David Pontille (2002).
} 
progressivement l'objet de la discipline, avec une prise en compte plus écologique des interactions :

«À l'origine centré sur l'organisation de la parole en interaction, il devient aujourd'hui organisé autour de l'« embodied interaction » (Streeck, Goodwin \& le Baron, 2010), définie par l'intrication entre des éléments vocaux, des orientations corporelles et des aspects liés à la matérialité de l'environnement. L'objectif de ces analyses consiste à repérer des configurations complexes de détails qui correspondent à des dimensions de l'organisation interactionnelle d'activités diverses. » (Relieu, 2013, p. 145)

\section{LES LIMITES DES PROMESSES DE LA NUMÉRISATION DE L'ENQUÊTE}

Le numérique n'est pas une révolution mais une étape de plus dans l'évolution des dispositifs d'enquête: par rapport aux autres médiations techniques, la numérisation affecte essentiellement le changement d'échelle (notamment au niveau du détail investigué ou dans la taille des corpus) et la circulation des données, offrant de nouvelles opportunités. Nous proposons d'en discuter ici trois: l'accès à de nouveaux terrains, les possibilités d'une collaboration accrue et enfin l'automatisation de la collecte d'un très grand nombre d'informations.

Première promesse de la numérisation: elle permettrait $\mathrm{d}^{\prime}$ accéder à de nouveaux terrains et de nouveaux objets. Si certains chercheurs et certaines chercheuses n'hésitent pas à avancer que le numérique équipe par exemple de nouvelles relations entre humains (Wesch, 2007) voire un nouveau processus d'individuation psychique et collective justifiant une "rupture anthropologique » (Puig et L'Hour, 2014), notre position est bien plus réservée sur le sujet. Â l'instar de nombreux et nombreuses collègues, nous sommes pour le moins sceptiques à l'endroit des discours mettant en avant toute forme de "révolution numérique », discours souvent empreint de déterminisme technologique. Comme Dominique Boullier (2016), nous pensons que le numérique ne fait qu'amplifier certains phénomènes déjà présents sous $\mathrm{d}^{\prime}$ autres formes et qu'il est fondamental de penser son enquête dès le départ pour ne pas écraser ces continuités. Ce danger guette dès qu'il y a une réification des mondes numériques (Pastinelli, 2011).

Pour autant, il est tout aussi faux de dire que le numérique n'a aucun effet: rares sont les activités humaines totalement à l'écart du numérique. Même une activité comme le tricot peut se faire "en ligne», au sens où des activités périphériques sur internet (recherche de patron, commande de laine, consultation de forum, poster une photo de son ouvrage terminé, etc.) vont venir accompagner la pratique du tricotage et ouvrir " au quotidien une fenêtre vers les espaces et les singularités des autres »(Zabban, 2016, p. 40). Quand bien même ces pratiques en ligne sont le fait d'une minorité de personnes, elles peuvent avoir des conséquences considérables: ici 
transformation du marché du tricot, de la distribution des savoirs et de la sociabilité autour de cette pratique de loisir (idem). S'il s'agit donc de ne pas isoler les activités numériques, on ne saurait pas plus invisibiliser les pans numériques de l'activité humaine. De ce fait, il faut se donner les moyens d'enquêter dessus.

Nous souhaitons apporter un dernier élément au débat sur la promesse d'objets nouveaux. En élargissant la palette des techniques d'enquêtes, les outils numériques permettent de recueillir des données inédites. En effet, certaines pratiques, parce que fugaces ou finement entrelacées, sont difficilement appréhendables par simple observation ou par entretien, notamment car elles ne sont pas toujours facilement perceptibles par les enquêtés. À titre d'exemple, citons les travaux déjà mentionnés d'Emmanuel Grimaud (2013), qui grâce à un oculomètre a permis de cartographier les mouvements des yeux et partant, d'apporter une contribution inédite à l'anthropologie de la vision. Plus largement, quand il s'agit d'analyser les usages des dispositifs numériques, par exemple l'usage d'un téléphone portable au cours d'une journée, les déclarations des usagers et usagères concernant la fréquence de leurs pratiques sont bien souvent en deçà des pratiques observées. Et parfois celles-ci sont tellement fugaces et répétées que l'observation directe avec consignation écrite s'avère difficilement tenable. Pour répondre à ces difficultés, à côté des techniques d'enquête relativement classiques, à l'instar du questionnaire administré en face à face (et sa version en ligne), des entretiens, de l'observation ou encore de l'analyse de sources de seconde main (articles de presse spécialisée, examen du code du logiciel, interviews, etc.), les sociologues des usages ont également développé des méthodologies innovantes comme le recueil de données de trafic pour un suivi des parcours des internautes, le recueil de l'activité sur un ordinateur par une sonde implantée ou des logiciels de capture d'écran (Beauvisage, 2007 ; Chaulet et Rouch, 2013), ou encore, à la suite des ergonomes et des ethnométhodologues, l'enregistrement vidéo de l'activité, parfois en situation de mobilité (grâce à des microcaméras intégrées à des badges ou des lunettes de soleil portées par l'enquêté).

Deuxième promesse : la numérisation via une simplification du partage à distance ouvre également de nouveaux horizons en matière de collaboration, pas seulement en termes de valorisation scientifique ${ }^{2}$, mais aussi pendant l'enquête, en permettant de collectiviser la collecte des données et leur analyse, voire après. En permettant de partager les données dès le début de leur collecte, et parfois même de façon synchrone, la numérisation présente de sérieux atouts en termes de partage des données de recherche, de collaboration entre chercheurs et d'administration de la preuve. Les carnets ethnographiques sur papier sont difficilement partageables pendant et après l'enquête (Datchary et Jeanjean, 2013). Leur version numérique, à l'instar des

\footnotetext{
${ }^{2}$ Comme cela a été annoncé dans l'introduction de cet article, nous n'aborderons pas cette question de la valorisation scientifique.
} 
carnets sur la plateforme Hypothèses (plateforme de blogs en sciences humaines et sociales ${ }^{3}$ ) ou des corpus partagés entre différents chercheurs et chercheuses sur des logiciels de type NVivo, permet une confrontation en continu à la communauté scientifique, et non plus seulement lors des conférences, journées d'étude, data sessions, etc. Elle permet également de se libérer en partie du formatage inhérent à ces exercices de présentation en public notamment en termes de longueur et de contenu. Le numérique permet non seulement d'enrichir le texte par la multiplication de notes, de liens, mais aussi de mélanger les médias (Dauphin et Derive, 2009) comme dans le cas des logiciels de vidéo annotée où ce n'est plus la transcription textuelle de la vidéo qui sert de base de référence à l'analyse, ou encore dans celui de stylos couplés $\mathrm{d}^{\prime}$ enregistreur et de scanner qui fournissent un document électronique où les notes prises par le chercheur ou la chercheuse sont couplées aux sons enregistrés. Dès lors, ces formats mêlant écrit et oral permettent à plusieurs logiques cognitives de coexister.

$\mathrm{Si}$, actualisées, ces potentialités constituent indéniablement une avancée pour la qualité du débat scientifique, il ne faut pas en sous-estimer les obstacles en termes d'administration de la preuve, via notamment la possibilité de réaliser des comparaisons dans le temps et dans l'espace ainsi que via la critique des pairs. Tout d'abord, un carnet de terrain en ligne occasionne un travail supplémentaire non négligeable, à moins d'imaginer une prise de notes directe ou le partage de données qui se présentent directement sous forme numérique. Par ailleurs, pour être exposé à la communauté scientifique, encore faut-il que sa production soit visible, et là encore une kyrielle de tâches supplémentaires se greffent pour obtenir un minimum de visibilité dans la communauté. Sans compter les compétences instrumentales à développer pour maîtriser ces outils de communication numérique. Ce n'est donc pas magique ni sans effort.

Dernière promesse et non des moindres : la numérisation rend possible un recueil de données à distance et automatisé. Si de fait de grands corpus peuvent être ainsi collectés, les anthropologues consciencieuses et consciencieux, sentant pointer plusieurs risques, ne resteront pas longtemps « assis » derrière leurs ordinateurs.

Tout d'abord, si de fait les corpus ainsi constitués peuvent être de taille imposante, on ne saurait toutefois céder à l'illusion de complétude des données. En effet, les données ainsi collectées ne sont que les traces d'une activité, le plus souvent incomplètes, car ces traces sont des données construites et formatées (Denis, 2018), dont il importe de déconstruire l'illusoire objectivité. À titre d'exemple, nous prendrons une enquête réalisée il y a plusieurs années sur les réseaux de jeunes altermondialistes (Datchary et Pagis, 2005). Une des questions que nous adressions à ce terrain était l'effectivité d'une plus grande horizontalité et de la logique du consensus dans les processus de prises de décision. Si nous nous étions contentées d'analyser

\footnotetext{
${ }^{3}$ https:/ / fr.hypotheses.org/
} 
les échanges de courriels et les échanges lors des réunions formelles, nous aurions raté un point essentiel dans le fonctionnement de ces collectifs, le poids décisif d'un petit groupe affinitaire. En effet, seule l'observation ethnographique par immersion nous a permis de montrer que derrière l'apparent fonctionnement au consensus sur l'espace numérique, voire en réunion formelle, d'autres échanges intervenaient par le truchement d'appels téléphoniques ou de rencontres informelles où les propositions étaient préparées par le même noyau de militants et militantes. Les personnes en désaccord avec ces propositions avaient beaucoup de mal à s'y opposer dans la mesure où, étant moins préparées et plus isolées, elles avaient rarement une alternative concrète à proposer. Si les données numériques ne sauraient à elles seules résumer l'activité, elles nécessitent en outre d'être nettoyées et codées, ce qui peut s'avérer extrêmement chronophage, à l'image de la retranscription de vidéos d'observation. Les traces récoltées sont d'une part complexes à interpréter telles quelles. Un travail plus ou moins long de (re-)codage est donc nécessaire. D'autre part, compte tenu de la taille des corpus, le temps d'analyse des données s'étend d'autant plus (Beauvisage, 2007). L'emploi de ces méthodes demande donc un travail de réflexivité important afin de décrire le processus de construction du dispositif, mais aussi d'expliciter les possibles biais introduits.

Et ce d'autant plus que la plupart des enquêtes qui exploitent ces traces se cantonnent souvent aux traces générées par une technologie particulière, voire une seule application ou fonctionnalité. Ce technocentrisme ou mediacentrisme n'est pas sans poser plusieurs problèmes. En premier lieu, il ne permet pas de restituer l'épaisseur des liens sociaux et la sociabilité numérique en train de se faire et la manière dont ils se structurent dans un contexte écologique communicationnel complexe, où les outils entretiennent des relations de chaînage, concurrence, interdépendance, etc. (Denis, 2003). Il paraît en effet plus judicieux de regarder l'écosystème technologique sur lequel est distribuée une activité et non pas les usages sociaux d'une technologie en particulier (Bailliencourt de, Beauvisage, Smoreda, 2007) ${ }^{4}$. De plus, ces entrées par un seul outil et/ou média génèrent une fragmentation des analyses, qui rend beaucoup plus difficile une capitalisation des savoirs. Enfin, cette démarche entretient les catégorisations a priori des outils, terreau du déterminisme technique. Quelle que soit la catégorisation opérée (synchrone/asynchrone, oral/écrit, etc.), elle tient rarement l'épreuve des usages (Broadbent, 2011 ; Velkovska 2004). Par exemple, un outil supposé asynchrone, comme le mail, peut faire l'objet d'un usage quasiment synchrone (Bretesché, de Corbière, Geffroy, 2014), ou encore que l'usage d'un outil écrit, comme la messagerie instantanée, peut davantage se rapprocher des modalités propres à l'oral (Licoppe, Cudicio, Proulx, 2011).

\footnotetext{
${ }^{4}$ Nous reviendrons sur ce point dans la section suivante.
} 


\section{RAISONNER LA NUMÉRISATION DE L'ENQUÊTE}

Comme nous venons de le voir, le numérique, loin de révolutionner l'enquête en sciences humaines et sociales, offre tout de même un certain nombre d'opportunités, à condition de prêter attention aux biais précédemment présentés. Pour donner à voir concrètement cette posture, nous allons mobiliser une enquête sur la communication au travail, sujet doublement pertinent au regard de la numérisation puisque celle-ci affecte tant l'objet étudié que les méthodes des chercheurs et chercheuses. En ce qui concerne l'objet, de fait, la prolifération de nouveaux moyens de communication permet d'entrer en contact avec davantage de personnes, tout en se jouant des barrières spatiales, rebattant ainsi les cartes des temporalités de l'activité travail (Datchary et Gaglio, 2014) mais aussi entre le travail et le hors travail. En découlent des questions en termes de multi-activité, d'interruptions ou de dispersion, pointant notamment les risques psychosociaux liés à ces situations (Datchary, 2011). Le terrain a été réalisé au sein d'un cabinet d'expertise comptable et d'audit de la région toulousaine. Nous ne présenterons pas plus en avant le métier d'experts-comptables dans la mesure où l'objet du propos ici n'est pas de présenter les résultats (Datchary et Cornillet, 2016), mais nos réflexions et arbitrages sur l'élaboration du protocole méthodologique. Retenons simplement, que comme dans la plupart des métiers, la communication y est importante tant entre collègues qu'avec les clients et clientes mais aussi les collaboratrices et collaborateurs externes (avocats et avocates, notaires, etc.).

$\mathrm{Si}$ nous avons pointé précédemment que de nombreuses activités comprenaient désormais un versant numérique, il ne faut pas oublier à l'inverse que les activités de communication ne se résument pas à leur part numérique. Nous avons donc fait le choix d'étudier la communication au travail en l'abordant en tant qu'activité à part entière. La communication ne se résume pas à une simple addition d'actes isolés, mais constitue bien une activité regroupant différentes formes de communication, qu'elles soient en face-à-face ou médiées par l'écrit ou la technique, synchrones ou asynchrones, mais aussi l'organisation des temporalités propres à chaque activité - ce qu'Anselm Strauss appelle « le travail d'articulation » (1992). Un point crucial pour les travailleurs et travailleuses est la gestion de leur disponibilité, du fait que cette activité communicationnelle multiforme peut entrer en conflit avec les autres activités de travail. Il s'agit donc de capter avec un maximum de détails l'ensemble des communications des personnes étudiées, ainsi que les contextes d'action dans lesquels ces communications prennent place.

Non seulement la communication se distribue sur différents artefacts, mais elle est également en partie imprévisible et entremêle différentes sphères du social. Ceci n'est pas sans poser des problèmes matériels et techniques pour «suivre » les usages tout au long de la chaîne technique et au cours des 
différentes activités. Aujourd'hui, ces problèmes tendent à s'amoindrir, et étudier l'activité communicationnelle, ou du moins l'enregistrer, devient plus «facile». Par exemple, les usages sur ordinateurs peuvent maintenant être appréhendés à partir de captures d'écran recueillies automatiquement (Chaulet et Rouch, 2013), voire déclenchées à l'initiative de l'enquêteur ou de l'enquêtrice. D'autres outils, notamment d'archivage, directement implantés dans les applications web peuvent également faciliter l'accès à certaines données. Cette relative facilité est une des raisons de la prolifération des enquêtes "médiacentrées » présentées précédemment. En raison des écueils déjà soulevés de telles démarches, nous avons opté pour appréhender l'usage entrelacé des différents outils communicationnels : il ne s'agit plus d'étudier des unités communicationnelles isolées, mais les types de relations dans lesquelles se (re)configurent ces unités (Bailliencourt de, Beauvisage, Smoreda, 2007).

Pour montrer toute la complexité d'un chaînage socio-technique, suivons par exemple une secrétaire qui se voit demander par le directeur du cabinet d'élaborer un dossier pour préparer un rendez-vous imminent. Devant accomplir cette tâche dans un délai de quinze minutes, la secrétaire suspend toutes ses activités en cours. Il lui faut rassembler divers documents que plusieurs collaborateurs et collaboratrices ont en leur possession. La première chose à faire est de déterminer qui possède quelle information, ou dans les termes de la secrétaire: "aller à la pêche à l'information ". Deux collaborateurs qu'il lui fallait solliciter se trouvaient dans des bureaux situés à l'opposé l'un de l'autre. Afin d'optimiser le temps, elle a passé un appel à la personne la plus éloignée de son bureau pour lui faire part de sa requête, a lancé des impressions dans l'open space, puis est allée solliciter en face-à-face la seconde personne. En retournant vers son bureau, passant devant le bureau d'une autre assistante, elle en a profité pour lui demander de l'aide. Elle a ensuite récupéré les documents imprimés, en a donné une partie à la deuxième assistante afin qu'elle fasse les reliures, et est retournée dans son bureau pour relier les autres documents. C'est alors que le premier collaborateur, celui contacté par téléphone, est venu dans son bureau pour lui demander si elle avait bien reçu son mail. Elle a alors suspendu les reliures pour aller consulter ses mails. Pendant ce temps, la personne lui a expliqué le contenu du mail, et indiqué qu'il n'avait pas lui non plus toutes les informations. Au même moment, le directeur est rentré dans le bureau pour s'enquérir de l'avancée du travail. La secrétaire l'informe alors des pièces manquantes, et les deux partent à la recherche de ces fameux documents. La scène a duré encore un certain temps (le délai initial de quinze minutes a été dépassé), et a continué de mobiliser différents outils et personnes. Une fois son travail accompli, elle est retournée à son bureau, a consulté ses mails, regardé ce qu'elle faisait avant cette activité, et collé un post-it sur son bureau en notant les tâches qu'elle n'avait pas eu le temps de faire en raison de cette interruption, afin de s'en souvenir le lendemain matin. 
Cet exemple issu du terrain permet de bien comprendre combien une tâche anodine de secrétariat peut recouvrir des actes communicationnels en cascade via différents médias. À la suite d'Olivier Martin (2013), nous estimons que mixer des méthodes est "nécessaire pour saisir l'entrelacement des outils de communication » dans la foisonnante variété de leurs formes. Afin de saisir sur le vif les communications au travail, nous avons élaboré un protocole méthodologique combinant observation directe, entretiens et recueil automatisé de traces de communication. Autrement dit, nous avons mixé des méthodes de captation digitale des données à des méthodes plus « classiques » d'ethnographie. L'enquête s'est déroulée en trois phases. Tout d'abord une phase de plusieurs mois d'observation ethnographique classique pour se familiariser avec l'entreprise et son organisation concrète du travail, pour ajuster le protocole de recueil des données afin qu'il corresponde au mieux aux pratiques effectives des personnes enquêtées tout en restant le moins invasif possible afin de ne pas troubler le déroulement "normal» des activités. Cette première phase de l'enquête a également été l'occasion de préparer la deuxième en déterminant huit personnes qui ont fait l'objet d'un suivi tout au long d'une (ou deux) journée(s) ${ }^{5}$. Ceci nous a permis d'effectuer un relevé systématique des actes communicationnels; un acte communicationnel étant défini comme toute forme de communication entre deux personnes ou plus, avec un échange sémantique qu'il soit oral ou écrit. Après un temps court de mise en forme et de première analyse de ces traces d'interactions, nous avons mené lors de la troisième phase des entretiens d'auto-confrontation avec six des huit enquêtés.

Pourquoi avoir procédé à une phase d'observation ethnographique préalable? Une première raison réside dans la négociation de l'accès au terrain, au niveau de l'organisation mais aussi des travailleurs et travailleuses. La participation au volet de suivi individuel de l'enquête s'est bien sûr faite sur la base du volontariat. Ce volet comprenait donc un dispositif de captation des données assez intrusif qui devait être compris et accepté par des personnes que nous souhaitions au profil assez divers, notamment au regard des fonctions occupées dans l'entreprise. Pour ce faire, il était nécessaire d'établir une relation de confiance elles, comme à chaque fois qu'il est question de pénétrer les territoires de l'intime. En effet, c'est bien l'activité numérique au travail, et non l'activité numérique de travail, dont il est question ici. Cette dernière s'entremêle avec de nombreuses activités relevant d'autres aspects de l'activité personnelle (communications avec la famille et plus largement l'entourage, achats en ligne, réseaux sociaux numériques, phases ludiques, etc.). Ce point permet également d'expliquer le peu d'enquêtes portant sur l'ensemble des moyens de communication. Certains outils permettent en effet de se passer de la relation avec les personnes enquêtées. Ainsi, dans de nombreuses enquêtes, le chercheur ou la chercheuse reste derrière son ordinateur et «siphonne » des données des utilisateurs et

\footnotetext{
${ }^{5}$ Soit au total 134 heures de suivi.
} 
utilisatrices à partir d'un outil - souvent Twitter - sans entrer en interaction avec la personne, voire sans même que cette dernière ne le sache. Pour notre enquête au contraire, nouer la relations de confiance mentionnée précédemment impliquait de passer du temps et d'interagir au préalable avec les membres du cabinet étudié.

Une seconde raison vient expliquer la nécessité d'une période d'observation prolongée pour une telle enquête. Passer plusieurs semaines à observer le fonctionnement et les interactions au sein de cette organisation professionnelle pour se familiariser avec l'activité de ses membres est une condition sine qua non pour ajuster le dispositif méthodologique initialement prévu aux spécificités de l'organisation enquêtée. En l'occurrence, étant donnée la confidentialité des informations échangées sur le terrain, qui, rappelons-le, est un cabinet d'expertise comptable, il ne nous a pas été possible d'obtenir ni l'accès direct aux écrans via une application de partage d'écran, ni l'autorisation d'installer de logiciel de capture automatique d'écran. Par ailleurs, cela a permis de définir correctement un panel judicieux de personnes à suivre et d'intégrer des dimensions non envisagées dans l'enquête. Cette préparation du dispositif en amont du suivi renforce la robustesse du corpus puisque la captation des traces n'est efficiente que si elle correspond à l'activité réelle observée.

Mais ce ne sont bien évidemment pas les seules raisons. Comme nous l'avons soulevé précédemment, un des biais inhérents aux traces numériques est le manque de contextualisation. Les données d'observation aident le chercheur ou la chercheuse à re-contextualiser les traces, et par là même, à éviter de tomber dans le piège d'une analyse trop rapidement menée qui nous ferait passer à côté d'aspects importants de l'activité communicationnelle. Prenons l'exemple de l'envoi d'un courriel. Si l'on ne fait que consulter l'historique des différentes boîtes de messagerie électronique, nous n'aurions accès qu'à l'heure d'envoi des e-mails, ce qui invisibilise tout le travail de préparation et de rédaction que nécessitent certains courriers électroniques. Pour notre enquête, l'observation a permis de compléter l'horodatage des actes communicationnels par un indicateur de durée, ce qui a participé à mettre en lumière une forte disparité concernant le temps de préparation des mails. L'observation a donc permis d'améliorer le versant numérique de l'enquête, mais aussi de pallier certains de ses défauts. En effet, un mail envoyé à un client ou une cliente peut nécessiter des échanges avec d'autres personnes du cabinet (comptables, responsables des fiches de paie, etc.). On touche ici à ce que nous avons mentionné précédemment: le chaînage communicationnel. Seuls, les historiques des boîtes de messagerie électronique ne permettent pas de mettre en lumière les relations entre ces actes communicationnels isolés artificiellement de par la disparité de leurs médiations, mais que l'observateur ou l'observatrice sur place aura tôt fait de rassembler. Par-delà l'apport de détails supplémentaires aux traces récoltées, l'observation nous permet d'accéder aux pratiques qui n'en laissent tout 
simplement pas. Lorsque l'on étudie la communication, il en est une dont on ne pourrait faire abstraction: la discussion en face-à-face. Le face-à-face, support premier de l'oralité, est souvent considéré comme un moyen noble de communication, notamment $\mathrm{du}$ fait qu'il se déroule en coprésence, situation supposée être la meilleure configuration pour une communication maîtrisée puisqu'elle met en jeu l'ensemble des sens des protagonistes. Le face-à-face reste la plupart du temps minimisé, voire invisibilisé, dans les enquêtes traitant de la numérisation de la communication au travail. Pourtant, les résultats de notre enquête montrent qu'en termes de durée, le face-à-face représente $58 \%{ }^{6}$ du temps total de communication observée. Concernant le volume, le face-à-face arrive en deuxième position, après le courriel, avec $34 \%{ }^{7}$. On ne saurait donc faire l'économie de l'étude de ce mode de communication, et pour cela, l'observation directe reste une méthode sans équivalent.

Mais qu'en est-il des traces? Pourquoi s'évertue-t-on à les capter si l'observation nous permet d'accéder à des données fines? Comme nous avons pu le voir, étudier finement l'activité communicationnelle dans sa foisonnante diversité requiert de porter son attention sur un large éventail de détails. Sur notre terrain par exemple, le nombre moyen d'actes communicationnels dans une journée de travail était de 140, avec des variations allant de 81 à 313 . Beaucoup d'échanges ne durent pas plus de quelques dizaines de secondes, certains moins, et d'autres même se superposent. De plus, souhaitant chronométrer chaque acte communicationnel, l'observation seule devenait insuffisante. Les limites des capacités humaines d'attention sont ici atteintes. Avec l'aide d'outils numériques, il est possible de maintenir un niveau d'observation flottante plus souhaitable pour une démarche inductive, et ainsi conserver une attention suffisante à l'environnement de la situation observée, tout en documentant finement l'ensemble des activités équipées. Nous avons donc intégré à notre protocole d'enquête la consultation régulière des historiques de l'ensemble des outils de communication observés. Ces traces d'activités permettent de documenter finement toutes les activités équipées, tout en gardant une attention suffisante pendant les observations, ce qui n'est pas négligeable lorsque l'on adopte une approche inductive comme nous l'avons fait. L'observation n'est pas la seule méthode que les données numériques permettent d'accompagner; les entretiens aussi peuvent être agrémentés de traces. Pensons notamment aux entretiens d'autoconfrontation (Datchary et Licoppe, 2007 ; Velkovska et Zouinar, 2007) qui nous permettent d'accéder à des représentations directes de l'activité, non plus spontanées, mais provoquées par la mobilisation des traces, saisissant ainsi le discours que tient la personne sur ses pratiques, les justifications qu'elle mobilise ainsi que le sens qu'elle donne à son action.

\footnotetext{
${ }^{6}$ Soit 3522 minutes de face-à-face pour 6044 minutes de communications observées.

7 Soit 641 actes en face-à-face pour un total de 1901 actes.
} 
Nous avons ainsi pu remarquer que le face-à-face n'était pas seulement minimisé par la littérature scientifique, mais également pas les travailleurs et travailleuses. En effet, à la question «Pouvez-vous me décrire votre communication? », ce mode de communication a le plus souvent été cité en dernier, sinon purement omis. Plusieurs raisons peuvent en partie expliquer cet état de fait. L'une d'elles tient aux correspondants et aux correspondantes. Pour certains et certaines, échanger avec les collègues ne relevait pas de la communication puisqu'ils concevaient cette dernière comme exclusivement dirigée vers l'extérieur de l'entreprise, ce qui les conduisait alors à minimiser le face-à-face. Les données relevées viennent appuyer cet argument, puisque $89 \%$ du volume d'actes communicationnels renseignés en tant que face-à-face ont été réalisés avec des collègues du cabinet. De plus, il est intéressant de noter que la majorité des face-à-face, soit $39 \%$, ont été relevés en tant que " discussion informelle ", c'est-à-dire comme non directement rattachés à une activité de travail, mais survenant soit aux différents moments de pauses dans la journée (café, déjeuner), soit à des rencontres fortuites lors de déplacements dans les locaux du cabinet. Ainsi, le fait que les échanges en face-à-face soient majoritairement des discussions informelles semble être une autre raison pour laquelle ce mode de communication est minimisé lors des entretiens. Or, bien que les sujets abordés lors de ces échanges ne soient pas directement liés au travail, il n'est pas rare que ce dernier surgisse dans le cours de la conversation. De plus, et ce point n'est pas négligeable, les discussions informelles au sein d'une organisation participent, notamment à travers le « support émotionnel » (Haythornthwaite et Wellman, 1998), à créer du lien en son sein, et ainsi à donner plus d'épaisseur et de stabilité aux collectifs de travail.

Un dernier exemple de ce que l'hybridation des méthodes d'observation et de collecte des traces nous a permis d'éclairer concerne la capacité d'agir des individus au travail, ou, pour reformuler, leurs méthodes face à l'épreuve de l'activité communicationnelle. De par leur perspective "médiacentrée », la plupart des enquêtes sur la communication au travail font état d'un isolement des travailleurs et travailleuses face à cette épreuve. Il apparait pourtant dans notre enquête que cette activité communicationnelle se distribue également sur le collectif de travail et son environnement via la négociation, modulation et publicisation de la disponibilité individuelle. C'est ainsi que nous avons pu observer un enquêté modifier à plusieurs reprises dans la même journée son statut sur le logiciel de messagerie instantanée du cabinet. Ses collègues qui souhaitent le contacter voient alors soit une icône verte ou rouge symbole de disponibilité ou, au contraire, d'indisponibilité, et décident de lui envoyer un message ou non, mais en connaissance de cause. Cette indication de disponibilité est également mobilisée pour d'autres types d'échanges. Avant d'aller solliciter ses collègues, ou de lui téléphoner, une enquêtée consulte presque systématiquement cet indicateur. Cet extrait de terrain n'est qu'un exemple parmi d'autres, mais que ce soit un casque audio posé sur la tête, une 
porte ouverte, mi-ouverte ou fermée, ou encore l'affichage de l'état de sa disponibilité via un post-it ou l'application de messagerie instantanée, ces tactiques sont variées en fonction du collectif de travail et des préférences individuelles. Toutes ces tactiques, ainsi que leurs significations pour celles et ceux qui les mettent en place, n'auraient pas pu être relevées si nous n'avions pas mobilisé à la fois l'observation, la récolte de traces d'activité et les entretiens.

\section{CONCLUSION}

L'examen que nous venons de faire de la numérisation de l'enquête en sciences humaines et sociales nous a conduit à faire un plaidoyer pour l'hybridation des méthodes numériques et «classiques». Nous avons tout d'abord procédé à un passage en revue des différentes médiations qui ont pu participer à transformer l'enquête. Ce faisant, nous avons pu voir que le numérique ne constitue pas une révolution, mais se trouve bien dans la continuité d'autres médiations. Que ce soit le corps, l'écriture, la photographie ou encore la vidéo, chacune de ces médiations contribue à modifier le rapport qu'entretient le chercheur ou la chercheuse avec son terrain, mais aussi le terrain lui-même. Il s'agit alors d'en prendre acte et d'expliciter les biais qui ont pu apparaitre lors de l'enquête. C'est ainsi que nous avons dans un deuxième temps abordé la numérisation du travail d'enquête en examinant trois de ses promesses : l'accès à de nouveaux terrains, la facilitation de la via le partage à distance sous des formats divers, le recueil des traces numériques d'activité de façon automatisée. Ces promesses ont toutes leurs limites propres que nous avons mises en lumière. La troisième partie constitue en quelque sorte l'application pratique des arguments développés dans les deux premières: nous y avons présenté en détail le dispositif méthodologique d'une enquête menée au sujet de l'activité communicationnelle, dispositif qui hybride méthodes "classiques » et numériques. Nous avons voulu mettre en évidence que, par un recours simultané et complémentaire de ces méthodes, nous pouvions pallier leurs biais respectifs. Si les méthodes numériques nous permettent la collecte d'une grande quantité de données avec un niveau fin de détail, les méthodes ethnographiques plus classiques nous permettent en amont d'adapter le dispositif pour qu'il soit au plus près de l'activité et pour contextualiser sur le moment ces données, ce qui offre en aval plus de robustesse à leur analyse. Par ailleurs, confronter en entretien les personnes enquêtées aux traces de leur activité permet d'analyser l'éventuel décalage qui peu, exister entre une activité et ses représentations, décalage le plus souvent heuristique. Ici en l'occurrence, il permet de pointer l'invisibilisation des communications de face-à-face.

En analysant l'activité communicationnelle au travail, nous avons montré combien ce serait une erreur de réduire celle-ci à son versant numérique, 
quand bien même ce dernier contribue sans conteste à modifier profondément les pratiques. Il en va de même quant à la numérisation de l'enquête, eu égard au travail de recherche en sciences humaines. Cette numérisation ne saurait révolutionner le travail d'enquête, au sens où les techniques plus classiques se trouveraient dépassées et progressivement abandonnées. Elle offre néanmoins des opportunités de recherche qu'il convient de saisir, en gardant à l'esprit qu'elle participe à modifier à la fois le rapport qu'entretient le chercheur ou la chercheuse avec son terrain et le terrain lui-même. Comme toute médiation technique, celle-ci a des incidences tant au niveau épistémologique qu'au niveau de la formation professionnelle au terrain, et notamment en termes de «méthodes » pour reprendre la terminologie de Garfinkel (1986), pour élargir au-delà de la question de la transmission des compétences visuelles, présentée dans la première partie de ce texte. Le développement de nouveaux agencements socio-techniques implique une période de latence avant la stabilisation de nouvelles méthodes reconnues et transmises au sein de la communauté scientifique. L'hybridation permet alors de se former progressivement à de nouvelles méthodes tout en gardant un haut niveau de réflexivité. Dans un contexte où les pratiques et la réflexivité ne sont pas encore stabilisées, il paraît en effet judicieux d'hybrider méthodes numériques et méthodes plus «classiques » pour en visualiser les écarts et mesurer leurs apports et faiblesses respectives.

\section{BIBLIOGRAPHIE}

Thomas BAILLIENCOURT De, Thomas BEAUVISAGE, Zbigniew SMOREDA, «La communication interpersonnelle face à la multiplication des technologies de contact », Réseaux, 145-146, 2007, pp.81-114.

Thomas BEAUVISAGE, "Mesurer l'activité sur ordinateur. Enjeux et méthodes », Réseaux, 6-7, 2007, pp.45-80.

Howard S. BECKER, «Sociologie visuelle, photographie documentaire et photojournalisme », Communications, 71, 2001, pp.333-351.

Dominique BOULLIER, Sociologie du numérique, Paris, Armand Colin, 2016.

Sophie BRETESCHÉ, François DE CORBIÈRE, Bénédicte GEFFROY, "Cadres et messageries », Réseaux, 187(5), 2014, pp.135-162.

Stefana BROADBENT, L'intimité au travail, Paris, FYP éditions, 2011.

Joël CANDAU et Agnès JEANJEAN, "Des odeurs à ne pas regarder... », Terrain. Anthropologie \& sciences humaines, 47, 2006, pp.51-68.

Antonio A. CASILLI, «Anthropologie et numérique: renouvellement méthodologique ou reconfiguration disciplinaire?", Anthrovision, 2.1, 2014, [En ligne]. URL : https://journals.openedition.org/anthrovision/626

Johann CHAULET et Jean Pierre ROUCH, «Le détail fait écran : capturer l'activité sur ordinateur ", in Caroline DATCHARY, Petit précis de méthodologie : le sens du détail dans les sciences sociales, Bordeaux, Le Bord de l'eau, 2013, pp. 71-89. 
Jean-Paul COLLEYN, « Champ et hors champ de l'anthropologie visuelle », L'Homme. Revue française d'anthropologie, 203-204(3), 2012, pp.457-480.

Eric DAGIRAL et Olivier MARTIN, L'ordinaire d'internet, Paris, Armand Colin, 2016.

Caroline DATCHARY, La dispersion au travail, Toulouse, Octarès, 2011.

Caroline DATCHARY, Petit précis de méthodologie: le sens du détail dans les sciences sociales, Bordeaux, Le bord de l'eau, 2013.

Caroline DATCHARY et Thomas CORNILLET, «Démêler l'écheveau de la communication au travail ", in Éric DAGIRAL et Olivier MARTIN (dir.), L'ordinaire d'internet, Paris, Armand Colin, 2016, pp.161-180.

Caroline DATCHARY et Gérald GAGLIO, «Hétérogénéité temporelle et activité de travail ", Revue d'anthropologie des Connaissances, 8, 2014, pp.1-22.

Caroline DATCHARY et Agnès JEANJEAN, "La place du détail dans les chaînes d'écriture de l'ethnographe ", in Caroline DATCHARY, Petit précis de méthodologie : le sens $d u$ détail dans les sciences sociales, Bordeaux, Le bord de l'eau, 2013, pp.153-169.

Caroline DATCHARY et Christian LICOPPE, «La multi-activité et ses appuis: l'exemple de la "présence obstinée" des messages dans l'environnement de travail. », @ctivités, 4(1), 2007, pp. 4-29, [En ligne]. URL : https://journals.openedition.org/activites/1370

Caroline DATCHARY et Julie PAGIS, « Jeunes altermondialistes en réseau », Réseaux, 23, 2005, pp.199-228.

Anne-Marie DAUPHIN et Jean DERIVE, « De quelques avatars de l'oralité littéraire », Parcours anthropologiques, 2009, pp.21-36, [En ligne]. URL: https://journals.openedition.org/pa/183

Jérôme DENIS, "La combinaison des TIC à l'interface de la relation-client dans les TPE », Réseaux, 21, 2003, pp.71-92.

Jérôme DENIS, Le travail invisible des données. Éléments pour une sociologie des infrastructures scripturales, Paris, Presses des Mines, 2018.

Jérôme DENIS et David PONTILLE, «L'écriture comme dispositif d'articulation entre terrain et recherche », Alinéa, Revue de Sciences Sociales et Humaines, 12, 2002, pp.93106.

Florence DUPRÉ, Rachel WALLISER, Joseph J. LÉVY, «Les ressources anthropologiques dans les cyberunivers: un aperçu ", Anthropologie et Sociétés, 35, 2011, pp.159-187.

Arturo ESCOBAR, «Welcome to Cyberia: Notes on the Anthropology of Cyberculture », Current anthropology, 35, 1994, pp.211-231.

Jeanne FAVRET-SAADA, «Être affecté », Gravidha, 8, 1990, pp.2-9.

Harold GARFINKEL, Ethnomethodological Studies of Work, Londres, Routledge, 1986.

Charles GOODWIN et Marjorie Harness GOODWIN, « Seeing as a Situated Activity: Formulating Planes ", in Yrjö ENGESTRÖM et David MIDDLETON (dir.), Cognition and communication at work, Cambridge, Cambridge University Press, 1996, pp. 61-95.

Jack GOODY, La raison graphique. La domestication de la pensée sauvage, Paris, Les Éditions de Minuit, 1979.

Emmanuel GRIMAUD, «Capter et mesurer les mouvements oculaires. Quels usages de l'oculométrie pour l'anthropologie de la vision? » in Caroline DATCHARY, (dir.) 
Le sens du détail. Petit précis de méthodologie en sciences sociales, Bordeaux, Les éditions du bord de l'eau, 2013, pp. 31-66.

Sylvie GROSJEAN, Florian GRANDENA, Luc BONNEVILLE, «Le chercheur, la caméra et l'organisation. Réflexion autour de la vidéo-ethnographie multi-située », SociologieS, 2017, [En ligne]. URL : https://journals.openedition.org/sociologies/6475 Caroline HAYTHORNTHWAITE et Barry WELLMAN, «Work, friendship, and media use for information exchange in a networked organization", Journal of the American Society for Information Science, 49(12), 1998, pp.1101-1114.

Edwin HUTCHINS, "Comment le "cockpit" se souvient de ses vitesses », Sociologie du travail, 4, 1994, pp.451-473.

Tim INGOLD, Marcher avec les dragons, Bruxelles, Zones sensibles, 2013.

Karin KNORR CETINA et Urs BRUEGGER, «Inhabiting Technology: The Global Lifeform of Financial Markets », Current sociology, 50, 2002, pp.389-405.

Muriel LEFEBVRE, "Les écrits scientifiques en action: pluralité des écritures et enjeux mobilisés », Sciences de la société, 67, 2006, pp.1-11.

L'Homme, Dossier « Littérature et anthropologie », vol. 29, n¹11-112, 1989.

Christian LICOPPE, Renato CUDICIO, Serge PROULX, "Présence connectée" au travail : les usages de la messagerie instantanée, le genre des "questions rapides" et l'économie morale de la "contribution" ", ethnographiques.org, 2011, [En ligne]. URL: https:// www.ethnographiques.org/2011/Licoppe-Cudicio-Proulx

Pierre LIVET, «Des actions et des émotions aux phénomènes sociaux : cognition et interprétation », Intellectica, 1-1, 1998, pp.57-77.

Olivier MARTIN, «Comment objectiver des pratiques et des usages ? », in Christine BARATS (dir.), Manuel d'analyse du web, Paris, Armand Colin, 2013, pp.223-277.

Luis Felipe Rosado MURILLO, "What Does “Open Data” Mean for Ethnographic Research?", American Anthropologist, 120, 2018, pp.577-582.

Madeleine PASTINELLI, « Pour en finir avec l'ethnographie du virtuel! Des enjeux méthodologiques de l'enquête de terrain en ligne », Anthropologie et sociétés, 35, 2011, pp.35-52.

Martine PERROT, Martin de la SOUDIÈRE (dir.), Dossier «L'écriture des sciences de l'homme ", Communication, 58, 1994.

Vincent PUIG et Yves-Marie L'HOUR, «Vers des nouveaux outils pour les Digital Studies », Anthropovision, 2, 2014, pp.2-18.

Marc RELIEU, «La transcription des détails configurants en Analyse Conversationnelle ", in Caroline DATCHARY (dir.), Petit précis de méthodologie. Le sens du détail dans les sciences sociales, Bordeaux, Le Bord de l'Eau, 2013, pp. 125-152.

Brian SCHWIMMER, "Anthropology on the internet: a review and evaluation of networked resources ", Current anthropology, 37, 1996, pp.561-568.

Anselm STRAUSS, La trame de la négociation. Sociologie qualitative et interactionnisme, Paris, L'Harmattan, 1992.

Julia VELKOVSKA, Les formes de la sociabilité électronique. Une sociologie des activités d'écriture sur internet, Thèse de doctorat de l'EHESS, Paris, 2004.

Julia VELKOVSKA et Moustafa ZOUINAR, «Interaction visiophonique et formes d'asymétries dans la relation de service », Réseaux, 25, 2007, pp.226-264. 
Michael WESCH, « What is Web 2.0 ? What Does It Mean for Anthropology ? Lessons From an Accidental Viral Video », Anthropology News, 48, 2007, pp.30-31.

Vinciane ZABBAN, «Tricoter en public. Internet et le "coming out" de la tricoteuse », in Éric DAGIRAL et Olivier MARTIN (dir.) L'ordinaire d'internet. Le web dans nos pratiques et relations sociales, Paris, Armand Colin, 2016, pp.35-57.

RÉSUMÉ : Nouveaux terrains d'investigation, nouveaux outils de récoltes de données, nouvelles pratiques de diffusion, la numérisation du travail ethnographique se présente souvent sous les auspices de la révolution. Pourtant ce n'est qu'une médiation supplémentaire du travail d'enquête ethnographique avec ses opportunités et ses contraintes. Dès lors, nous proposons dans cet article de l'envisager dans une logique de complément aux méthodes déjà existantes plutôt que de substitution. Pourquoi et comment articuler les méthodes ? Cette double question sera d'abord envisagée d'un point de vue théorique, puis traitée concrètement à travers les arbitrages réalisés dans le dispositif méthodologique d'une enquête récente sur la communication au travail.

MOTS-CLÉS : Numérisation, Ethnographie, Enquête, Réflexivité, Méthodologie 